\title{
A Mock Theta Function of Second Order
}

\author{
Bhaskar Srivastava \\ Department of Mathematics and Astronomy, Lucknow University, Lucknow 226 007, India \\ Correspondence should be addressed to Bhaskar Srivastava, bhaskarsrivastav@yahoo.com
}

Received 10 September 2009; Accepted 31 December 2009

Recommended by Rodica Costin

We consider the second-order mock theta function $\Phi_{5}(q)$, which Hikami came across in his work on mathematical physics and quantum invariant of three manifold. We give their bilateral form, and show that it is the same as bilateral third-order mock theta function of Ramanujan. We also show that the mock theta function $\Phi_{5}(q)$ outside the unit circle is a theta function and also write $h_{1}(q)$ as a coefficient of $z^{0}$ of a theta series. First writing $h_{1}(q)$ as a coefficient of a theta function, we prove an identity for $h_{1}(q)$.

Copyright (C) 2009 Bhaskar Srivastava. This is an open access article distributed under the Creative Commons Attribution License, which permits unrestricted use, distribution, and reproduction in any medium, provided the original work is properly cited.

\section{Brief History of Mock Theta Functions}

The mock theta functions were introduced and named by Ramanujan and were the subjects of Ramanujan's last letter to Hardy, dated January 12, 1920, to be specific [1, 2, pages 354355]. Ramanujan gave a list of seventeen functions which he called "mock theta functions." He divided them into four groups of functions of order 3, 5, 5, and 7. Ramanujan did not rigorously define a mock theta function nor he define the order of a mock theta function. A definition of the order of a mock theta function is given in the Gordon-McIntosh paper on modular transformation of Ramanujan's fifth and seventh-order mock theta functions [3] Watson [4] while constructing transformation laws for the mock theta function found three further mock theta functions of order 3.

In 1976, Andrews while visiting Trinity college, Cambridge, discovered in the mathematical library of the college a notebook written by Ramanujan towards the end of his life and Andrews called it "Lost" Notebook. In the lost notebook were six more mock theta functions and linear relation between them. Andrews and Hickerson [5] called these mock theta functions of sixth-order and proved the identities.

In the "Lost" Notebook on page 9 appear four more mock theta functions which were called by Choi of tenth-order. Ramanujan also gave eight linear relations connecting these mock theta functions of tenth-order and these relations were proved by Choi [6]. 
Gordon and McIntosh listed eight functions in their eighth-order paper [7], but later, in their survey paper [8], classified only four of them as eighth-order. The other four are more simple in their modular transformation laws and therefore are considered to be of lower order.

We now come to the second-order mock theta functions. McIntosh [9] considered three second-order mock theta functions and gave transformation formulas for them. Hikami [10] in his work on mathematical physics and quantum invariant of three manifold came across the $q$-series:

$$
\begin{aligned}
\Phi_{5}(q) & =\sum_{n=0}^{\infty} \frac{q^{n}(-q ; q)_{n}}{\left(q ; q^{2}\right)_{n+1}} \\
& =\frac{1}{\left(q ; q^{2}\right)_{\infty}^{2}} \sum_{n=0}^{\infty}\left(q ; q^{2}\right)_{n}^{2} q^{2 n}
\end{aligned}
$$

and proved that $\Phi_{5}(q)$ is a mock theta function and called it of " 2 nd" order.

He further showed that $\Phi_{5}(q)$ is a sum of two mock theta functions $h_{1}(q)$ and $\omega(q)$ where $h_{1}(q)$ is of second-order and $\omega(q)$ is Ramanujan's mock theta function of third-order. This $\Phi_{5}(q)$ will be the basis of our study in this paper.

Before we begin with the study of $\Phi_{5}(q)$ and $h_{1}(q)$ it will be appropriate to mention the work done earlier.

Gordon and McIntosh in their survey paper [8] have shown that $h_{1}(q)$ is essentially the odd part of the second-order mock theta function $B(q)$, which appears as $\beta(q)$ in Andrews' paper on Mordell integrals and Ramanujan's lost notebook [11] and also in McIntosh paper on second-order mock theta functions [9]. In particular,

$$
h_{1}\left(q^{2}\right)=\frac{B(q)-B(-q)}{4 q}
$$

where

$$
B(q)=\sum_{n=0}^{\infty} \frac{q^{n(n+1)}\left(-q^{2} ; q^{2}\right)_{n}}{\left(q ; q^{2}\right)_{n+1}^{2}}=\sum_{n=0}^{\infty} \frac{q^{n}\left(-q ; q^{2}\right)_{n}}{\left(q ; q^{2}\right)_{n+1}}
$$

Since the even part of $B(q)$ is the ordinary theta function

$$
\frac{B(q)+B(-q)}{2}=\left(q^{4} ; q^{4}\right)_{\infty}\left(-q^{2} ; q^{2}\right)_{\infty}^{4}
$$

it follows that the odd part and $h_{1}(q)$ are second-order mock theta functions. Thus $\Phi_{5}(q)$ is a linear combination of second-order and third-order mock theta function. In some sense, mock theta functions of orders 1, 2, 3, 4, and 6 are all in the same family.

The paper is divided as follows.

In Section 3 we expand $\Phi_{5}(q)$ as a bilateral $q$-series and show that it is also a sum of the second-order mock theta function $\Phi_{5}(q)$ and the third-order mock theta function $\omega(q)$. 
By using Bailey's transformation we have the interesting result that the bilateral $\Phi_{5, c}(q)$ is the same as the bilateral $\omega_{c}(q)$.

In Section 4 , using bilateral transformation of Slater, we write $\Phi_{5, c}(q)$ as a bilateral series ${ }_{2} \psi_{2}$ series with a free parameter $c$.

In Section 5 , a mild generalization $\Phi_{5, c}(z, \alpha)$ of $\Phi_{5, c}(q)$ is given and we show that this generalized function is a $F_{q}$-function.

In Section 6 we show that $\Phi_{5}(q)$, outside the unit circle $|q|=1$, is a theta function.

In Section 7 we state a generalized Lambert Series expansion for $h_{1}(q)$ as given in [8].

In Section 8 we show that $h_{1}(q)$ is a coefficient of $z^{0}$ of a theta function. function.

In Section 9 we prove an identity for $h_{1}(q)$ using $h_{1}(q)$ as a coefficient of $z^{0}$ of a theta method.

In Section 10 a double series expansion for $h_{1}(q)$ is obtained by using Bailey pair

\section{Basic Preliminaries}

We first introduce some standard notation.

If $q$ and $a$ are complex numbers with $|q|<1$ and $n$ is a nonnegative integer, then

$$
\begin{gathered}
(a)_{0}=(a ; q)_{0}=1 \\
(a)_{n}=(a ; q)_{n}=\prod_{k=0}^{n-1}\left(1-a q^{k}\right), \\
(a)_{\infty}=(a ; q)_{\infty}=\prod_{k=0}^{\infty}\left(1-a q^{k}\right), \\
\left(a_{1}, \ldots, a_{m}\right)_{n}=\left(a_{1}, \ldots, a_{m} ; q\right)_{n}=\left(a_{1} ; q\right)_{n}, \ldots,\left(a_{m} ; q\right)_{n} .
\end{gathered}
$$

Ramanujan's mock theta function of third-order $\omega(q)$ and $v(q)$ is

$$
\begin{gathered}
\omega(q)=\sum_{n=0}^{\infty} \frac{q^{2 n(n+1)}}{\left(q ; q^{2}\right)_{n+1}^{2}}, \\
v(q)=\sum_{n=0}^{\infty} \frac{q^{n(n+1)}}{\left(-q ; q^{2}\right)_{n+1}}, \\
\phi(q)=\sum_{n=-\infty}^{\infty} q^{n^{2}}=\left(-q ; q^{2}\right)_{\infty}^{2}\left(q^{2} ; q^{2}\right)_{\infty}=\frac{(-q ;-q)_{\infty}}{(q ;-q)_{\infty}} .
\end{gathered}
$$

We will use the following notations for $\theta$-functions.

Definition 2.1. If $|q|<1$ and $x \neq 0$, then

$$
j(x, q)=\left(x, \frac{q}{x}, q ; q\right)_{\infty} .
$$


If $m$ is a positive integer and $a$ is an integer,

$$
\begin{gathered}
J_{a, m}=j\left(q^{a}, q^{m}\right), \\
\bar{J}_{a, m}=j\left(-q^{a}, q^{m}\right), \\
J_{m}=j\left(q^{m}, q^{3 m}\right)=\left(q^{m} ; q^{m}\right)_{\infty^{\prime}} \\
j\left(\frac{q}{x}, q\right)=j(x, q), \\
j(x, q)=-x j\left(x^{-1}, q\right), \\
j\left(q^{n} x, q\right)=(-1)^{n} q^{-n(n-1) / 2} x^{-n} j(x, q), \quad \text { if } n \text { is an integer. }
\end{gathered}
$$

By Jacobi's triple product identity [12, page 282]

$$
j(x, q)=\sum_{n=-\infty}^{\infty}(-1)^{n} q^{n(n-1) / 2} x^{n} .
$$

\subsection{More Definitions}

If $z$ is a complex number with $|z| \neq 1$, then

$$
\varepsilon(z)= \begin{cases}1 & \text { if }|z|<1 \\ -1 & \text { if }|z|>1\end{cases}
$$

If $s$ is an integer, then

$$
\operatorname{sg}(s)= \begin{cases}1 & \text { if } s \geq 0 \\ -1 & \text { if } s<0\end{cases}
$$

Using these definitions,

$$
\frac{1}{1-z}=\varepsilon(z) \sum_{\substack{s=-\infty \\ \operatorname{sg}(s)=\varepsilon(z)}}^{\infty} z^{s}
$$

We shall use the following theorems.

Theorem 2.2 (see [13, Theorem 1.3, page 644]). Let $q$ be fixed, $0<|q|<1$. Let $a, b$, and $m$ be fixed integers with $b \neq 0$ and $m \geq 1$. Define

$$
F(z)=\frac{1}{j\left(q^{a} z^{b}, q^{m}\right)}
$$


Then $F$ is meromorphic for $z \neq 0$, with simple poles at all points $z_{0}$ such that $z_{0}^{b}=q^{k m-a}$ for some integer $k$. The residue of $F(z)$ at such a point $z_{0}$ is

$$
\frac{(-1)^{k+1} q^{m k(k-1) / 2} z_{0}}{b J_{m}^{3}}
$$

Theorem 2.3 (see [13, Theorem 1.8(a), page 647]). Suppose that

$$
F(z)=\sum_{r} F_{r} z^{r}
$$

for all $z \neq 0$ and that $F(z)$ satisfies

$$
F(q z)=C z^{-n} F(z)
$$

where $0<|q|<1$ and $C \neq 0$. Then

$$
F(z)=\sum_{r=0}^{n-1} F_{r} z^{r} j\left(-C^{-1} q^{r} z^{n}, q^{n}\right) .
$$

Truesdell [14] calls the functions which satisfy the difference equation

$$
\frac{\partial}{\partial z} F(z, \alpha)=F(z, \alpha+1)
$$

as F-function. He unified the study of these F-functions.

The functions which satisfy the q-analogue of the difference equation

$$
D_{q, z} F(z, \alpha)=F(z, \alpha+1)
$$

where

$$
z D_{q, z} F(z, \alpha)=F(z, \alpha)-F(z q, \alpha)
$$

are called $F_{q}$-functions.

\section{Bilateral $\Phi_{5}(q)$ as a Sum of Two Mock Theta Functions of Different Orders}

(i) We shall denote the bilateral of $\Phi_{5}(q)$ by $\Theta_{5, c}(q)$. We define it as

$$
\left(q ; q^{2}\right)_{\infty}^{2} \Phi_{5, c}(q)=\sum_{n=-\infty}^{\infty}\left(q ; q^{2}\right)_{n}^{2} q^{2 n}
$$


Now

$$
\begin{aligned}
\left(q ; q^{2}\right)_{\infty}^{2} \Phi_{5, c}(q) & =\sum_{n=-\infty}^{\infty}\left(q ; q^{2}\right)_{n}^{2} q^{2 n} \\
& =\sum_{n=0}^{\infty}\left(q ; q^{2}\right)_{n}^{2} q^{2 n}+\sum_{n=-1}^{-\infty}\left(q ; q^{2}\right)_{n}^{2} q^{2 n} \\
& =\sum_{n=0}^{\infty}\left(q ; q^{2}\right)_{n}^{2} q^{2 n}+\sum_{n=0}^{\infty} \frac{q^{2 n^{2}+2 n}}{\left(q ; q^{2}\right)_{n+1}^{2}}
\end{aligned}
$$

and we use (1.2) in the first summation and (2.2) in the second summation, to write

$$
\left(q ; q^{2}\right)_{\infty}^{2} \Phi_{5, c}(q)=\left(q ; q^{2}\right)_{\infty}^{2} \Phi_{5}(q)+\omega(q)
$$

Thus $\Phi_{5, c}(q)$ is a sum of a second-order mock theta function and a third-order mock theta function.

(ii) Transformation of Bilateral $\Phi_{5, c}(q)$ into bilateral $\omega_{c}(q)$ is as follows.

It is very interesting that the bilateral $\Phi_{5, c}(q)$ can be written as bilateral third-order mock theta function $\omega_{c}(q)$.

We use Bailey's bilateral transformation [15, 5.20(ii), page 137]:

$$
{ }_{2} \psi_{2}\left[\begin{array}{l}
a, b \\
c, d
\end{array} ; q, z\right]=\frac{(a z, b z, c q / a b z, d q / a b z ; q)_{\infty}}{(q / a, q / b, c, d ; q)_{\infty}} \times{ }_{2} \psi_{2}\left[\begin{array}{c}
a b z / c, a b z / d \\
a z, b z
\end{array} ; q, c d / a b z\right] .
$$

Letting $q \rightarrow q^{2}$, and setting $a=b=q, c=d=0$, and $z=q^{2}$ in (3.4), we get

$$
\left(q ; q^{2}\right)_{\infty}^{2} \Phi_{5, c}(q)=\frac{\left(q^{3}, q^{3} ; q^{2}\right)_{\infty}}{\left(q, q ; q^{2}\right)_{\infty}} \sum_{n=-\infty}^{\infty} \frac{q^{2 n^{2}+2 n}}{\left(q^{3} ; q^{2}\right)_{n}^{2}}=\sum_{n=-\infty}^{\infty} \frac{q^{2 n^{2}+2 n}}{\left(q ; q^{2}\right)_{n+1}^{2}}=\omega_{c}(q) .
$$

\section{Another Bilateral Transformation}

Slater [15, (5.4.3), page 129] gave the following transformation formula, and we have taken $r=2$ :

$$
\begin{aligned}
& \frac{\left(b_{1}, b_{2}, q / a_{1}, q / a_{2}, d z, q / d z ; q\right)_{\infty}}{\left(c_{1}, c_{2}, q / c_{1}, q / c_{2} ; q\right)_{\infty}} \psi_{2}\left[\begin{array}{l}
a_{1}, a_{2} \\
b_{1}, b_{2} ; q, z
\end{array}\right] \\
& \quad=\frac{q}{c_{1}} \frac{\left(c_{1} / a_{1}, c_{1} / a_{2}, q b_{1} / c_{1}, q b_{2} / c_{1}, d c_{1} z / q, q^{2} / d c_{1} z ; q\right)_{\infty}}{\left(c_{1}, q / c_{1}, c_{1} / c_{2}, q c_{2} / c_{1} ; q\right)_{\infty}} \psi_{2}\left[\begin{array}{l}
q a_{1} / c_{1}, q a_{2} / c_{1} \\
q b_{1} / c_{1}, q b_{2} / c_{1}
\end{array} ; q, z\right] \\
& \quad+\operatorname{idem}\left(c_{1} ; c_{2}\right)
\end{aligned}
$$


where $d=a_{1} a_{2} / c_{1} c_{2},\left|b_{1} b_{2} / a_{1} a_{2}\right|<|z|<1$, and idem $\left(c_{1} ; c_{2}\right)$ after the expression means that the preceding expression is repeated with $c_{1}$ and $c_{2}$ interchanged.

In the transformation it is interesting that the $c^{\prime}$ s are absent in the ${ }_{2} \psi_{2}$ series on the left side of (4.1). This gives us the freedom to choose the $c^{\prime} s$ in a convenient way.

Letting $q \rightarrow q^{2}$ and setting, $a_{1}=a_{2}=q, b_{1}=b_{2}=0$, and $z=q^{2}$ in (4.1), so $d=q^{2} / c_{1} c_{2}$ and $0<|z|<1$, to get

$$
\begin{aligned}
& \frac{\left(q ; q^{2}\right)_{\infty}^{4}\left(q^{4} / c_{1} c_{2} ; q^{2}\right)_{\infty}\left(c_{1} c_{2} / q^{2} ; q^{2}\right)_{\infty}}{\left(c_{2} ; q^{2}\right)_{\infty}\left(q^{2} / c_{2} ; q^{2}\right)_{\infty}} \Phi_{5, c}(q) \\
& \quad=\frac{q^{2}}{c_{1}} \frac{\left(c_{1} / q ; q^{2}\right)_{\infty}^{2}\left(q^{2} / c_{2} ; q^{2}\right)_{\infty}\left(c_{2} ; q^{2}\right)_{\infty}}{\left(c_{1} / c_{2} ; q^{2}\right)_{\infty}\left(q^{2} c_{2} / c_{1} ; q^{2}\right)_{\infty}} \sum_{n=-\infty}^{\infty}\left(q^{3} / c_{1} ; q^{2}\right)_{n}^{2} q^{2 n}+\operatorname{idem}\left(c_{1} ; c_{2}\right) .
\end{aligned}
$$

By choosing $c_{1}$ suitably we can have different expansion identities. Moreover (4.2) can be seen as a generalization of (3.3).

\section{Mild Generalization of $\Phi_{5, c}(q)$}

We define the bilateral generalized function $\Phi_{5, c}(z, \alpha)$ as

$$
\left(q ; q^{2}\right)_{\infty}^{2} \Phi_{5, c}(z, \alpha)=\frac{1}{(z)_{\infty}} \sum_{n=-\infty}^{\infty}\left(q ; q^{2}\right)_{n}^{2}(z)_{n} q^{n \alpha+n}
$$

For $\alpha=1, z=0, \Phi_{5, c}(z, \alpha)$ reduce to $\Phi_{5, c}(q)$.

Now

$$
\begin{aligned}
D_{q, z}\left[\Phi_{5, c}(z, \alpha)\right] & =\frac{1}{z}\left[\Phi_{5, c}(z, \alpha)-\Phi_{5, c}(z q, \alpha)\right] \\
& =\frac{1}{z\left(q ; q^{2}\right)_{\infty}^{2}}\left[\frac{1}{(z)_{\infty}} \sum_{n=-\infty}^{\infty}\left(q ; q^{2}\right)_{n}^{2}(z)_{n} q^{n \alpha+n}-\frac{1}{(z q)_{\infty}} \sum_{n=-\infty}^{\infty}\left(q ; q^{2}\right)_{n}^{2}(z q)_{n} q^{n \alpha+n}\right] \\
& =\frac{1}{z\left(q ; q^{2}\right)_{\infty}^{2}} \frac{1}{(z)_{\infty}} \sum_{n=-\infty}^{\infty}\left(q ; q^{2}\right)_{n}^{2}(z)_{n} q^{n \alpha+n}\left(1-\left(1-z q^{n}\right)\right) \\
& =\frac{1}{\left(q ; q^{2}\right)_{\infty}^{2}(z)_{\infty}} \sum_{n=-\infty}^{\infty}\left(q ; q^{2}\right)_{n}^{2}(z)_{n} q^{n \alpha+2 n} \\
& =\Phi_{5, c}(z, \alpha+1) .
\end{aligned}
$$

So $\Phi_{5, c}(z, \alpha+1)$ is an $F_{q}$-function. 

following.

Being $F_{q}$-function it has unified properties of $F_{q}$-functions. For example, one has the

(i) The inverse operator $D_{q, x}^{-1}$ of $q$-differentiation is related to $q$-integration as

$$
D_{q, x}^{-1} \phi(x)=(1-q)^{-1} \int \phi(x) d_{q} x
$$

See Jackson [16].

(ii) $D_{q, z}^{n} F_{q}(z, \alpha)=F_{q}(z, \alpha+n)$, where $n$ is a nonnegative integer.

\section{Behaviour of $\Phi_{5}(q)$ outside the Unit Circle}

By definition (1.1)

$$
\Phi_{5}(q)=\sum_{n=0}^{\infty} \frac{(-q ; q)_{n}}{\left(q ; q^{2}\right)_{n+1}} q^{n}
$$

Replacing $q$ by $1 / q$ and writing $\Phi_{5}^{*}(q)$ for $\Phi_{5}(1 / q)[10]$,

$$
\begin{aligned}
\boldsymbol{\Xi}_{5}^{*}(q) & =\sum_{n=0}^{\infty} \frac{(-1)^{n} q^{\left(n^{2}+n\right) / 2}(-q ; q)_{n}}{\left(q ; q^{2}\right)_{n+1}} \\
& =1-q^{2}+q^{6}-q^{12}+q^{20}-q^{30}+\cdots \\
& =\sum_{n=0}^{\infty}(-1)^{n} q^{n^{2}+n}
\end{aligned}
$$

which is a $\theta$-function.

\section{Lambert Series Expansion for $h_{1}(q)$}

For the double series expansion, we first require the generalized Lambert series expansion for $h_{1}(q)$.

By Entry 12.4.5, of Ramanujan's Lost Notebook [17, page 277], Hikami [10] noted that

$$
\Phi_{5}(q)=2 h_{1}(q)-(-q ; q)_{\infty}^{2} \omega(q)
$$

where

$$
h_{1}(q)=\sum_{n=0}^{\infty} \frac{(-q ; q)_{2 n}}{\left(q ; q^{2}\right)_{n+1}^{2}} q^{n} .
$$

There is a slight misprint in the definition $h_{1}(q)$ in Hikami's paper [10] which has been corrected and Gordon and McIntosh have also pointed out in their survey [8]. 
International Journal of Mathematics and Mathematical Sciences

In [8] the Lambert series expansion for $h_{1}(q)$ is

$$
\begin{aligned}
h_{1}(q) & =\sum_{n=0}^{\infty} \frac{(-q ; q)_{2 n}}{\left(q ; q^{2}\right)_{n+1}^{2}} q^{n} \\
& =\frac{1}{\theta_{4}(0, q)} \sum_{n=0}^{\infty} \frac{(-1)^{n} q^{n(n+2)}}{1-q^{2 n+1}} \\
& =\frac{1}{2 \theta_{4}(0, q)} \sum_{n=-\infty}^{\infty} \frac{(-1)^{n} q^{n(n+2)}}{1-q^{2 n+1}} .
\end{aligned}
$$

\section{8. $h_{1}(q)$ as a Coefficient of $z^{0}$ of a $\theta$-Function}

In the following theorem of Hickerson [13, Theorem 1.4, page 645],

$$
\sum_{r=-\infty}^{\infty} \frac{x^{r}}{1-q^{r} y}=\frac{J_{1}^{3} j(x y, q)}{j(x, q) j(y, q)}
$$

let $q \rightarrow q^{2}$, and then put $y=q$, to get

$$
\sum_{r=-\infty}^{\infty} \frac{x^{r}}{1-q^{2 r+1}}=\frac{J_{2}^{3} j\left(q x, q^{2}\right)}{j\left(x, q^{2}\right) j\left(q, q^{2}\right)} .
$$

For $|q|<1$, and $z \neq 0$ and not an integral power of $q$, let

$$
A(z)=\frac{1}{2 \theta_{4}(0, q)} \frac{J_{2}^{3} j\left(q z, q^{2}\right)}{j\left(z, q^{2}\right) j\left(q, q^{2}\right)} j\left(\frac{z}{q}, q^{2}\right) .
$$

Theorem 8.1. Let $q$ be fixed with $0<|q|<1$. Then $h_{1}(q)$ is the coefficient of $z^{0}$ in the Laurent series expansion of $A(z)$ in the annulus $|q|<|z|<1$.

Proof. By (7.3)

$$
\begin{aligned}
2 \theta_{4}(0, q) h_{1}(q) & =\sum_{n=-\infty}^{\infty} \frac{(-1)^{n} q^{n(n+2)}}{1-q^{2 n+1}} \\
& =\text { coefficient of } z^{0} \text { in } \sum_{n=-\infty}^{\infty} \frac{z^{n}}{1-q^{2 n+1}} \sum_{s=-\infty}^{\infty}(-1)^{s} q^{s^{2}+s}\left(\frac{z}{q}\right)^{-s} \\
& =\text { coefficient of } z^{0} \text { in } \frac{J_{2}^{3} j\left(q z, q^{2}\right)}{j\left(z, q^{2}\right) j\left(q, q^{2}\right)} j\left(\frac{z}{q}, q^{2}\right)
\end{aligned}
$$

dividing by $2 \theta_{4}(0, q)$ gives the theorem. 


\section{An Identity for $h_{1}(q)$}

Theorem 9.1. If $0<|q|<1$ and $z$ is neither zero nor an integral power of $q$, then

$$
\begin{aligned}
A(z, q)= & j\left(z, q^{2}\right) h_{1}(q) \\
& -\left(-\frac{1}{2}\right) \sum_{r=-\infty}^{\infty} \frac{(-1)^{r} q^{r^{2}+3 r-1} z^{r+2}}{1-q^{2 r+2} z} \\
& -\frac{1}{2} \sum_{r=-\infty}^{\infty} \frac{(-1)^{r} q^{r^{2}+3 r+1} z^{-r-1}}{1-q^{2 r+2} z^{-1}} .
\end{aligned}
$$

Define

$$
\begin{gathered}
L(z)=-\frac{1}{2} \sum_{r=-\infty}^{\infty} \frac{(-1)^{r} q^{r^{2}+3 r-1} z^{r+2}}{1-q^{2 r+2} z} \\
M(z)=\frac{1}{2} \sum_{r=-\infty}^{\infty} \frac{(-1)^{r} q^{r^{2}+3 r+1} z^{-r-1}}{1-q^{2 r+2} z^{-1}}, \\
F(z)=A(z)+L(z)+M(z) .
\end{gathered}
$$

The scheme will be first to show that $F(z)$ satisfies the functional relation:

$$
F\left(q^{2} z\right)=-z^{-1} F(z)
$$

One considers the poles of $L(z)$ and $M(z)$ and shows that the residue of $F(z)$ at these poles is zero. So $F(z)$ is analytic at these points. One then shows that the coefficients of $z^{0}$ in $L(z)$ and $M(z)$ are zero and equating the coefficient of $z^{0}$ in (9.4) one has the theorem.

Proof. We show that

$$
F\left(q^{2} z\right)=-z^{-1} F(z)
$$

We shall show that each of $A(z), L(z)$, and $M(z)$ satisfies the functional equation:

$$
A(z)=\frac{1}{2} \frac{(-q ; q)_{\infty}}{(q ; q)_{\infty}} \frac{J_{2}^{3} j\left(q z, q^{2}\right)}{j\left(z, q^{2}\right) j\left(q, q^{2}\right)} j\left(\frac{z}{q}, q^{2}\right),
$$


and so

$$
A\left(q^{2} z\right)=\frac{1}{2} \frac{(-q ; q)_{\infty}}{(q ; q)_{\infty}} \frac{J_{2}^{3} j\left(q^{3} z, q^{2}\right)}{j\left(q^{2} z, q^{2}\right) j\left(q, q^{2}\right)} j\left(z q, q^{2}\right) .
$$

We employ (2.11) on the right-hand side to get

$$
\begin{gathered}
A\left(q^{2} z\right)=\frac{1}{2} \frac{(-q ; q)_{\infty}}{(q ; q)_{\infty}} \frac{J_{2}^{3}(-1) z^{-1} q^{-1} j\left(z q, q^{2}\right)(-1) q z^{-1}}{(-1) z^{-1} j\left(z, q^{2}\right) j\left(q, q^{2}\right)} j\left(\frac{z}{q}, q^{2}\right) \\
A\left(q^{2} z\right)=-z^{-1} A(z) .
\end{gathered}
$$

We now take $L(z)$ :

$$
L\left(q^{2} z\right)=\frac{1}{2} \sum_{r=-\infty}^{\infty} \frac{(-1)^{r} q^{r^{2}+3 r-1}\left(z q^{2}\right)^{r+2}}{1-q^{2 r+2}\left(z q^{2}\right)}
$$

Writing $r-1$ for ron the right-hand side we have

$$
L\left(q^{2} z\right)=-z^{-1} L(z)
$$

Similarly only writing $r+1$ for $r$ we have

$$
M\left(q^{2} z\right)=-z^{-1} M(z)
$$

Hence the functional equation (9.4) is proved.

Obviously $L(z)$ and $M(z)$ are meromorphic for $z \neq 0 . L(z)$ has simple poles at $z=q^{2 k-2}$ and $M(z)$ has simple poles at $z=q^{2 k+2}$. Hence $F(z)$ is meromorphic for $z \neq 0$ with, at most, simple poles at $z=q^{2 k \pm 2}$.

Taking $r=0$ in (9.2), we calculate the residue of $L(z)$ at the point $z=1 / q^{2}$ :

$$
\text { Residue of } L(z)=\lim _{z \rightarrow 1 / q^{2}} \frac{1}{2}\left(z-\frac{1}{q^{2}}\right) \frac{z^{2} q^{-1}}{\left(z-\left(1 / q^{2}\right)\right) q^{2}}=\frac{1}{2} q^{-5}
$$


For the residue of $A(z)$ at $z=1 / q^{2}$, take $b=1, k=-1, m=2, a=0$ in (2.16) to get

$$
\text { Residue of } \begin{aligned}
A(z) & =\frac{1}{2} \frac{(-q ; q)_{\infty}}{(q ; q)_{\infty}} \frac{J_{2}^{3} j\left(1 / q, q^{2}\right) j\left(1 / q^{3}, q^{2}\right)}{j\left(q, q^{2}\right)} \frac{1}{J_{2}^{3}} \\
& =\frac{1}{2} \frac{(-q ; q)_{\infty}}{(q ; q)_{\infty}} \frac{j\left(q, q^{2}\right) j\left(q^{3}, q^{2}\right)}{q^{4} j\left(q, q^{2}\right)} \\
& =\frac{1}{2} \frac{(-q ; q)_{\infty}}{(q ; q)_{\infty}} \frac{1}{q^{4}}\left(q^{3} ; q^{2}\right)_{\infty}\left(\frac{1}{q} ; q^{2}\right)_{\infty}\left(q^{2} ; q^{2}\right)_{\infty} \\
& =\frac{1}{2} \frac{(-q ; q)_{\infty}}{(q ; q)_{\infty}} \frac{1}{q^{4}} \frac{(1-(1 / q))}{(1-q)}\left(q ; q^{2}\right)_{\infty}\left(q ; q^{2}\right)_{\infty}\left(q^{2} ; q^{2}\right)_{\infty} \\
& =-\frac{1}{2} \frac{(-q ; q)_{\infty}}{(q ; q)_{\infty}} \frac{1}{q^{5}} \frac{(q ; q)_{\infty}}{(-q ; q)_{\infty}} \\
& =-\frac{1}{2} q^{-5} .
\end{aligned}
$$

So the residue of $F(z)$ at $z=1 / q^{2}$ is $-(1 / 2) q^{5}+0+(1 / 2) q^{5}=0$.

Now we calculate the residue at $z=q^{2}$ :

$$
\text { Residue of } \begin{aligned}
M(z) & =\lim _{z \rightarrow q^{2}} \frac{1}{2}\left(z-q^{2}\right) \frac{q z^{-1}}{\left(1-q^{2} z^{-1}\right)} \\
& =\lim _{z \rightarrow q^{2}} \frac{1}{2}\left(z-q^{2}\right) \frac{q}{\left(z-q^{2}\right)} \\
& =\frac{q}{2}
\end{aligned}
$$

and for the residue of $A(z)$ at $z=q^{2}$, taking $b=1, k=1, m=2$, and $a=0$ in (2.16), so

$$
\text { Residue of } \begin{aligned}
A(z) & =\frac{1}{2} \frac{(-q ; q)_{\infty}}{(q ; q)_{\infty}} \frac{J_{2}^{3} j\left(q^{3}, q^{2}\right) j\left(q, q^{2}\right)}{j\left(q, q^{2}\right)} \frac{q^{2}}{J_{2}^{3}} \\
& =\frac{1}{2} \frac{(-q ; q)_{\infty}}{(q ; q)_{\infty}} j\left(q^{3}, q^{2}\right) q^{2} \\
& =\frac{1}{2} \frac{(-q ; q)_{\infty}}{(q ; q)_{\infty}}\left(q^{3} ; q^{2}\right)_{\infty}\left(\frac{1}{q} ; q^{2}\right)_{\infty}\left(q^{2} ; q^{2}\right)_{\infty} q^{2} \\
& =\frac{1}{2} \frac{(-q ; q)_{\infty}}{(q ; q)_{\infty}} \frac{(1-(1 / q))}{(1-q)}\left(q ; q^{2}\right)_{\infty}\left(q ; q^{2}\right)_{\infty}\left(q^{2} ; q^{2}\right)_{\infty} q^{2} \\
& =-\frac{1}{2} q .
\end{aligned}
$$

Hence the residue of $F(z)$ at $z=q^{2}$ is $0+(1 / 2) q-(1 / 2) q=0$. Hence $F(z)$ is analytic at $z=q^{2}$. 
Since $F(z)$ satisfies (9.4), so $F(z)$ is analytic at all points of the form $z=q^{2 k \pm 2}$ and hence for all $z \neq 0$.

We now apply (2.20) with $n=1$ and $c=-1$ and $q$ replaced by $q^{2}$ to get

$$
F(z)=F_{0} j\left(z, q^{2}\right)
$$

where $F_{0}$ is the coefficient of $z^{0}$ in the Laurent expansion of $F(z), z \neq 0$.

Now for $|q|<|z|<1$, by Theorem 8.1, the coefficient of $z^{0}$ in $A(z)$ is $h_{1}(q)$.

For such $z,\left|q^{2 r+2} z\right|<1$ if and only if $r \geq 0$.

That is,

$$
\varepsilon\left(q^{2 r+2} z\right)=\operatorname{sg}(r)
$$

Hence by (2.15)

$$
\frac{1}{1-q^{2 r+2} z}=\operatorname{sg}(r) \sum_{\substack{r=-\infty \\ \operatorname{sg}(r)=\operatorname{sg}(s)}}^{\infty} q^{(2 r+2) s} z^{s} .
$$

So

$$
L(z)=-\frac{1}{2} \sum_{\operatorname{sg}(r)=\operatorname{sg}(s)}^{\infty} \operatorname{sg}(r)(-1)^{r} q^{r^{2}+3 r-1+(2 r+2) s} z^{r+2+s} .
$$

If $\operatorname{sg}(r)=\operatorname{sg}(s)$, then $r+s+2$ is either $\geq 1$ or $\leq-1$; so coefficient of $z^{0}$ in $L(z)$ is 0 . Similarly the coefficient of $z^{0}$ in $M(z)$ is 0 and so the coefficient of $z^{0}$ in $F(z)$ is $h_{1}(q)$.

Hence by (9.17), we have

$$
F(z)=h_{1}(q) j\left(z, q^{2}\right)
$$

which gives the theorem.

\section{Double Series Expansion}

Now we derive the double series expansion for $h_{1}(q)$. We shall use the Bailey pair method, as used by Andrews [18] for fifth and seventh-order mock theta functions and by Andrews and Hickerson [5] for sixth-order mock theta functions.

We define Bailey pair.

Two sequences $\left\{\alpha_{n}\right\}$ and $\left\{\beta_{n}\right\}, n \geq 0$, form a Bailey pair relative to a number $a$ if

$$
\beta_{n}=\sum_{r=0}^{n} \frac{\alpha_{r}}{(q)_{n-r}(a q)_{n+r}}
$$

for all $n \geq 0$. 
Corollary 10.1 (see [5, Corollary. 2.1, page 70]). If $\left\{\alpha_{n}\right\}$ and $\left\{\beta_{n}\right\}$ form a Bailey pair relative to $a$, then

$$
\sum_{n=0}^{\infty} \frac{\left(\rho_{1}\right)_{n}\left(\rho_{2}\right)_{n}\left(a q / \rho_{1} \rho_{2}\right)^{n} \alpha_{n}}{\left(a q / \rho_{1}\right)_{n}\left(a q / \rho_{2}\right)_{n}}=\frac{(a q)_{\infty}\left(a q / \rho_{1} \rho_{2}\right)_{\infty}}{\left(a q / \rho_{1}\right)_{\infty}\left(a q / \rho_{2}\right)_{\infty}} \sum_{n=0}^{\infty}\left(\rho_{1}\right)_{n}\left(\rho_{2}\right)_{n}\left(\frac{a q}{\rho_{1} \rho_{2}}\right)^{n} \beta_{n}
$$

provided that both sums converge absolutely.

We state the theorem of Andrews and Hickerson [5, Theorem 2.3, pages 72-73].

Let $a, b, c$, and $q$ be complex numbers with $a \neq 1, b \neq 0, c \neq 0, q \neq 0$, and none $a / b, a / c$, $q b, q c$ of the form $q^{-k}$ with $k \geq 0$. For $n \geq 0$, define

$$
\begin{aligned}
A_{n}^{\prime}(a, b, c, q)= & \frac{q^{n^{2}}(b c)^{n}\left(1-a q^{2 n}\right)(a / b)_{n}(a / c)_{n}}{(1-a)(q b)_{n}(q c)_{n}} \\
& \times \sum_{j=0}^{n} \frac{(-1)^{j}\left(1-a q^{2 j-1}\right)(a)_{j-1}(b)_{j}(c)_{j}}{\left.q^{j}\right)(b c)^{j}(q)_{j}(a / b)_{j}(a / c)_{j}}, \\
B_{n}^{\prime}(a, b, c, q)= & \frac{1}{(q b)_{n}(q c)_{n}} .
\end{aligned}
$$

Then the sequences $\left\{A_{n}^{\prime}(a, b, c, q)\right\}$ and $\left\{B_{n}^{\prime}(a, b, c, q)\right\}$ form a Bailey pair relative to $a$.

Letting $q \rightarrow q^{2}$ and then taking $a=q^{2}, b=c=q$, in (10.3), we get

$$
\begin{aligned}
A_{n}^{\prime}\left(q^{2}, q, q^{2}\right)= & \frac{q^{2 n^{2}+2 n}\left(1-q^{4 n+2}\right)\left(q ; q^{2}\right)_{n}^{2}}{\left(1-q^{2}\right)\left(q^{3} ; q^{2}\right)_{n}^{2}} \\
& \times \sum_{j=0}^{n} \frac{(-1)^{j}\left(1-q^{4 j}\right)\left(q^{2} ; q^{2}\right)_{j-1}\left(q ; q^{2}\right)_{j}^{2}}{q^{j^{2}+j}\left(q^{2} ; q^{2}\right)_{j}\left(q ; q^{2}\right)_{j}^{2}} \\
= & \frac{(1-q)^{2}\left(1+q^{2 n+1}\right) q^{2 n^{2}+2 n}}{\left(1-q^{2}\right)\left(1-q^{2 n+1}\right)} \sum_{j=0}^{n}(-1)^{j} q^{-j^{2}-j}\left(1+q^{2 j}\right) \\
= & \frac{(1-q)^{2}\left(1+q^{2 n+1}\right) q^{2 n^{2}+2 n}}{\left(1-q^{2}\right)\left(1-q^{2 n+1}\right)}\left[1+\sum_{j=-n}^{n}(-1)^{j} q^{-j^{2}-j}\right], \\
B_{n}^{\prime}\left(q^{2}, q, q, q^{2}\right)= & \frac{1}{\left(q^{3} ; q^{2}\right)_{n}^{2}} .
\end{aligned}
$$

Now letting $q \rightarrow q^{2}$ and then setting $\rho_{1}=-q, \rho_{2}=-q^{2}, a=q^{2}$ in (10.2) we get

$$
\sum_{n=0}^{\infty} \frac{q^{n}\left(-q ; q^{2}\right)_{n}}{\left(-q^{3} ; q^{2}\right)_{n}} \alpha_{n}=\frac{\left(q ; q^{2}\right)_{\infty}\left(q^{4} ; q^{2}\right)_{\infty}}{\left(-q^{2} ; q^{2}\right)_{\infty}\left(-q^{3} ; q^{2}\right)_{\infty}} \sum_{n=0}^{\infty}\left(-q ; q^{2}\right)_{n}\left(-q^{2} ; q^{2}\right)_{n} q^{n} \beta_{n}
$$


Taking $A_{n}^{\prime}$ and $B_{n}^{\prime}$ for $\alpha_{n}^{\prime}$ and $\beta_{n}^{\prime}$, respectively, in (10.5) and using the definition of $h_{1}(q)$, we get

$$
\frac{\left(q ; q^{2}\right)_{\infty}\left(q^{2} ; q^{2}\right)_{\infty}}{\left(-q ; q^{2}\right)_{\infty}\left(-q^{2} ; q^{2}\right)_{\infty}} h_{1}(q)=\sum_{n=0}^{\infty} \frac{q^{2 n^{2}+3 n}}{1-q^{2 n+1}}\left[1+\sum_{j=-n}^{n}(-1)^{j} q^{-j^{2}-j}\right]
$$

or

$$
h_{1}(q)=\frac{\left(-q ; q^{2}\right)_{\infty}\left(-q^{2} ; q^{2}\right)_{\infty}}{\left(q ; q^{2}\right)_{\infty}\left(q^{2} ; q^{2}\right)_{\infty}} \sum_{n=0}^{\infty} \frac{q^{2 n^{2}+3 n}}{1-q^{2 n+1}}\left[1+\sum_{j=-n}^{n}(-1)^{j} q^{-j^{2}-j}\right]
$$

which is the double series expansion for $h_{1}(q)$.

This double series expansion can be used to get more properties of $\nexists_{5}(q)$.

\section{References}

[1] S. Ramanujan, Collected Papers, Cambridge University Press, Cambridge, UK, 1927.

[2] S. Ramanujan, Collected Papers, Chelsea, New York, NY, USA, 1962.

[3] B. Gordon and R. J. McIntosh, "Modular transformations of Ramanujan's fifth and seventh order mock theta functions," The Ramanujan Journal, vol. 7, pp. 193-222, 2003.

[4] G. N. Watson, "The final problem: an account of the mock theta functions," Journal of the London Mathematical Society, vol. 11, pp. 55-80, 1936.

[5] G. E. Andrews and D. Hickerson, "Ramanujan's "lost" notebook. VII. The sixth order mock theta functions," Advances in Mathematics, vol. 89, no. 1, pp. 60-105, 1991.

[6] Y.-S. Choi, "Tenth order mock theta functions in Ramanujan's lost notebook. IV," Transactions of the American Mathematical Society, vol. 354, no. 2, pp. 705-733, 2002.

[7] B. Gordon and R. J. McIntosh, "Some eighth order mock theta functions," Journal of the London Mathematical Society, vol. 62, no. 2, pp. 321-335, 2000.

[8] B. Gordon and R. J. McIntosh, "A survey of classical mock theta functions," preprint.

[9] R. J. McIntosh, "Second order mock theta functions," Canadian Mathematical Bulletin, vol. 50, no. 2, pp. 284-290, 2007.

[10] K. Hikami, "Transformation formula of the "second" order mock theta function," Letters in Mathematical Physics, vol. 75, no. 1, pp. 93-98, 2006.

[11] G. E. Andrews, "Mordell integrals and Ramanujan's lost notebook," in Analytic Number Theory, Lecture Notes, vol. 899, pp. 10-48, Springer, Berlin, 1981.

[12] G. H. Hardy and E. M. Wright, An Introduction to the Theory of Numbers, Oxford University Press, London, UK, 4th edition, 1968.

[13] D. Hickerson, "A proof of the mock theta conjectures," Inventiones Mathematicae, vol. 94, no. 3, pp. 639-660, 1988.

[14] C. Truesdell, An Essay Toward a Unified Theory of Special Functions, Annals of Mathematics Studies, no. 18, Princeton University Press, Princeton, NJ, USA, 1948.

[15] G. Gasper and M. Rahman, Basic Hypergeometric Series, vol. 35 of Encyclopedia of Mathematics and Its Applications, Cambridge University Press, Cambridge, UK, 1990.

[16] F. H. Jackson, "Basic integration," Quart. J. Math. (Oxford), (2), vol. 2, pp. 1-16, 1951.

[17] G. E. Andrews and B. C. Berndt, Ramanujan's Lost Notebook, Part I, Springer, New York, NY, USA, 2005.

[18] G. E. Andrews, "The fifth and seventh order mock theta functions," Transactions of the American Mathematical Society, vol. 293, no. 1, pp. 113-134, 1986. 


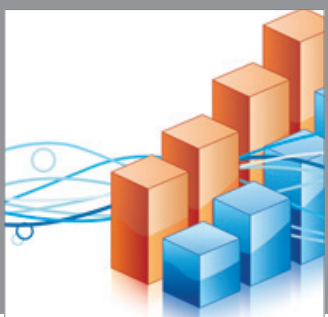

Advances in

Operations Research

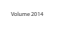

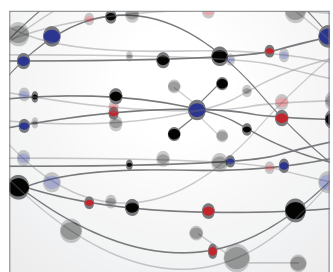

\section{The Scientific} World Journal
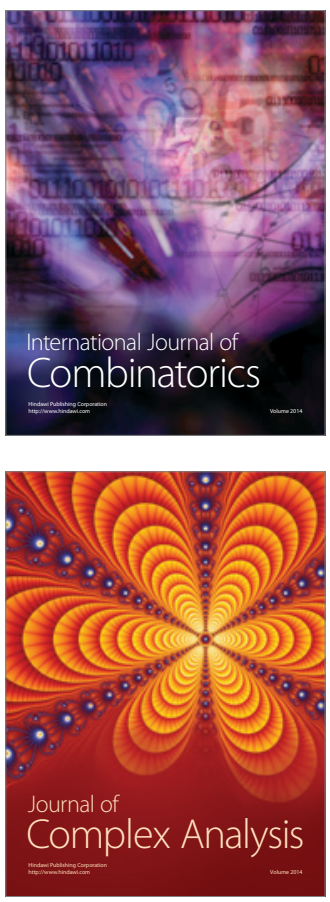

International Journal of

Mathematics and

Mathematical

Sciences


Journal of

Applied Mathematics
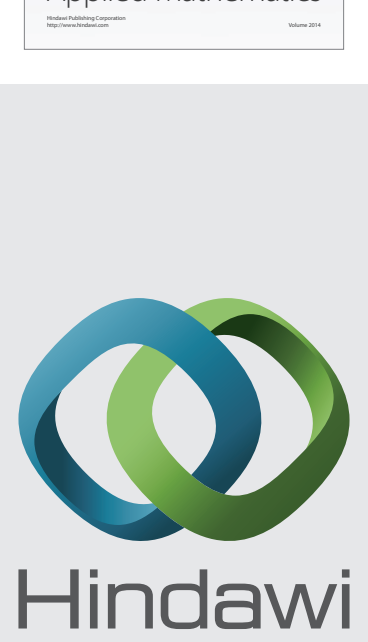

Submit your manuscripts at http://www.hindawi.com


Mathematical Problems in Engineering
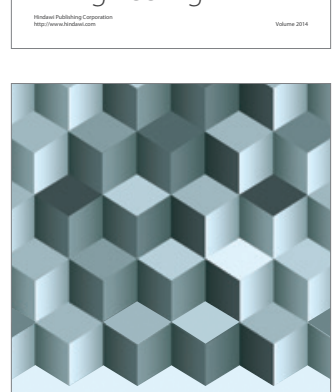

Journal of

Function Spaces
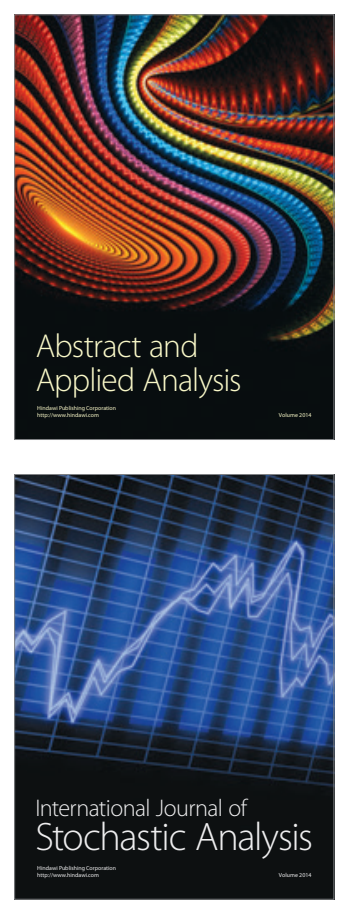



ournal of

Probability and Statistics

Promensencen
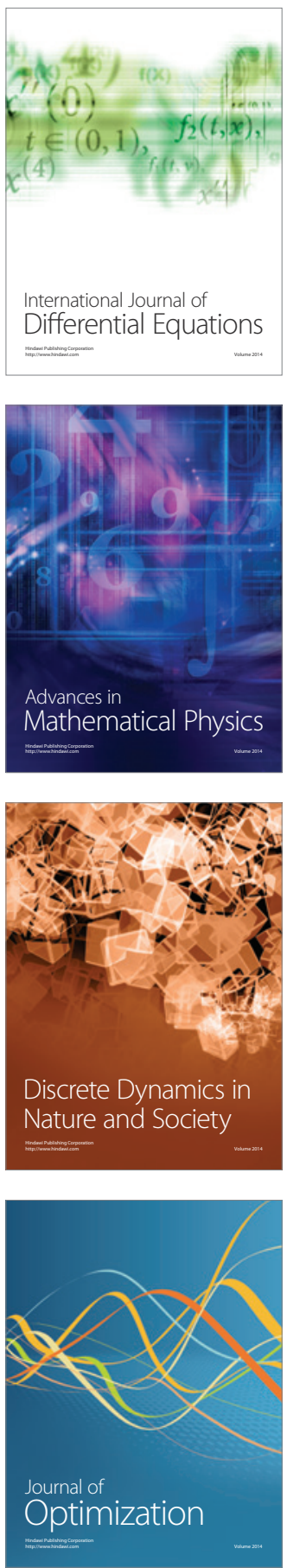